\title{
THE COMPLETE SYMMETRY GROUP OF A FORCED HARMONIC OSCILLATOR
}

\author{
P. G. L. LEACH
}

(Received 14 June 1979)

(Revised 10 July 1979)

\begin{abstract}
The complete symmetry group of a forced harmonic oscillator is shown to be $S I(3, R)$ in the one-dimensional case. Approaching the problem through the Hamiltonian invariants and the method of extended Lie groups, the method used is that of time-dependent point transformations. The result applies equally well to the forced repulsive oscillator and a particle moving under the influence of a coordinate-free force. The generalization to $n$-dimensional systems is discussed.
\end{abstract}

\section{Introduction}

Over the last few years there has been a resurgence of interest in the symmetries of dynamical systems. The original impetus for the construction of dynamical symmetry groups came from the description of unexpected degeneracies in spectra. For the basic integrable classical systems, the Kepler problem and the harmonic oscillator, the symmetry groups are respectively $S O(4)$ and $S U(3)$ (for three dimensions). Similarities between time-independent and time-dependent oscillator systems led to the study of the invariants, and consequently the symmetries, of time-dependent quadratic Hamiltonians. It was seen that time-dependent and time-independent quadratic Hamiltonians were related by linear canonical transformations [5]. Previously known invariants [4] for the time-independent oscillator were shown to have their counterparts for time-dependent oscillators $[3,6]$. In particular, the Lewis invariant [12] was given a simple explanation. The existence of these invariants enabled the construction of symmetry groups for quadratic Hamiltonians [7]. As the invariants usually had non-zero Poisson brackets with the Hamiltonian, they were called non-invariance symmetry groups.

Further work [9] on quadratic Hamiltonians showed that there are more linear 
and quadratic invariants than is necessary to form the operators of the special unitary group. The significance of these invariants became obvious in light of the work of Anderson and Davison [1], Wulfman and Wybourne [17], and Lutzky [13]. Anderson and Davison showed that the complete symmetry group for the onedimensional time-independent harmonic oscillator and the free particle was $S l(3, R)$. The term complete is used to indicate that the group is the largest admitted by the problem. Wulfman and Wybourne used the method of extended Lie groups to deduce the same result for the oscillator. Lutzky used Noether's theorem and a modification of the theory of extended Lie groups to repeat the result.

The extension of these results to other systems has been undertaken recently. Leach has shown that $S l(3, R)$ applies to the one-dimensional time-dependent harmonic oscillator [10] and to the repulsive oscillator [11]. Of the eight generators required, five came from the two linear and three quadratic invariants of the Hamiltonian and the remaining three from the use of the method of extended Lie groups. Prince and Eliezer [15] extended the work on the time-dependent oscillator to $n$-dimensions, showing that the group is $S l(n+2, R)$. They followed Lutzky's method.

In this note, the complete symmetry group of a forced one-dimensional harmonic oscillator is shown to be $S l(3, R)$. The method used is based on the theory of linear canonical transformations. Lutzky's approach has not been attempted, but it is expected that the results would be equally easy to obtain. Some work was done using the method of Wulfman and Wybourne. The resulting partial differential equations were not easy to solve and it is believed that the method adopted here is simpler. It also has the advantage of showing the essential similarity of linear Hamiltonian systems.

\section{Lie symmetry groups and invariants}

The main ideas of the theory of Lie symmetry groups may be found in Bluman and Cole [2]. Some of the relevant results are summarized here for the benefit of the reader. A one-parameter infinitesimal point transformation from coordinates $(q, t)$ to coordinates $(\bar{q}, \bar{t})$, where

$$
\bar{t}=t+\xi(q, t) \delta \alpha, \quad \bar{q}=q+\eta(q, t) \delta \alpha,
$$

is generated by the operator

$$
G(q, t) \equiv \xi(q, t) \frac{\partial}{\partial t}+\eta(q, t) \frac{\partial}{\partial q} .
$$

To find the variations induced on the derivatives it is necessary to use the extended 
group operator. For a function involving the first derivative, the operator is

$$
G^{(1)} \equiv \xi \frac{\partial}{\partial t}+\eta \frac{\partial}{\partial q}+\eta^{(1)} \frac{\partial}{\partial \dot{q}}
$$

and, for one involving the second derivatives, it is

where

$$
G^{(2)} \equiv \xi \frac{\partial}{\partial t}+\eta \frac{\partial}{\partial q}+\eta^{(1)} \frac{\partial}{\partial \dot{q}}+\eta^{(2)} \frac{\partial}{\partial \ddot{q}}
$$

$$
\left.\begin{array}{l}
\eta^{(1)} \equiv \frac{d \eta}{d t}-\dot{q} \frac{d \xi}{d t}, \\
\eta^{(2)} \equiv \frac{d \eta^{(1)}}{d t}-\ddot{q} \frac{d \xi}{d t}, \\
\frac{d}{d t} \equiv \frac{\partial}{\partial t}+\dot{q} \frac{\partial}{\partial q} .
\end{array}\right\}
$$

In particular, if it is desired to study the symmetry group of a differential equation of the second order, such as

$$
N(q, \dot{q}, \ddot{q}, t)=0,
$$

the second extended operator must be used. An operator $G$ is said to be the generator of a one-parameter symmetry group for (2.6) if, whenever (2.6) is satisfied,

$$
\begin{gathered}
G^{(2)} N(q, \dot{q}, \ddot{q}, t)=0 \\
\Leftrightarrow \xi \frac{\partial N}{\partial t}+\eta \frac{\partial N}{\partial q}+\eta^{(1)} \frac{\partial N}{\partial \dot{q}}+\eta^{(2)} \frac{\partial N}{\partial \ddot{q}}=0 .
\end{gathered}
$$

It is possible to obtain the generator of a one-parameter symmetry group if the motion corresponding to the differential equation (2.6) has an integral invariant. In terms of the Lagrangian formulation, if there exists an invariant, it may be written in the form

$$
\phi(q, \dot{q}, t)=(\xi q-\eta) \frac{\partial L}{\partial \dot{q}}-\xi L+f(q, t) .
$$

The operator $G$ is obtained by the use of the $\xi$ and $\eta$ which satisfies (2.9) for a particular $\phi(q, \dot{q}, t)$. In the Hamiltonian formulation the corresponding relation is

$$
\phi(q, p, t)=\xi H-\eta p+f(q, t) .
$$

The function $f(q, t)$ performs the task of soaking up remaining terms. For each invariant $\phi$ there corresponds only one generator. It has been noted by Lutzky [14] that, were $f$ allowed to depend upon $q, \dot{q}, t$ (or $q, p, t$ ), this uniqueness property 
is lost. From (2.10) it is obvious that, for those Hamiltonians which are at most quadratic in the momentum, invariants are also at most quadratic in the momentum if they are to give rise to a symmetry group generator.

It should be emphasized that, although to each invariant (subject to the limitations mentioned above) there exists a generator of a one-parameter Lie group, there may be one-parameter groups which do not correspond to the Lagrangian (or Hamiltonian) invariants. The generators of such groups are formed from the solutions of (2.8). They have the property of leaving the equation of motion invariant, but not the action integral. Only the Lagrangian (Hamiltonian) derived generators have this property. There has been some work done on finding constants which correspond to these additional generators, but so far the results have not been reported in the literature [16].

\section{Invariants for the forced oscillator}

The one-dimensional, time-independent, forced harmonic oscillator has Newtonian equation of motion

$$
+\ddot{q} q+f=0,
$$

where $f$ is taken to be a continuous function of time over the interval. of time which is of interest. The corresponding Hamiltonian is

$$
H=\frac{1}{2}\left(p^{2}+q^{2}\right)+f q,
$$

the conjugate momentum, $p$, being $\dot{q}$. This may be transformed to the unforced oscillator

$$
\bar{H}=\frac{1}{2}\left(P^{2}+Q^{2}\right)
$$

by means of a linear canonical transformation. Rewriting (3.2) and (3.3) as

$$
\begin{gathered}
H=\frac{1}{2} \mathbf{z}^{\mathrm{T}} I \mathbf{z}+\mathbf{b}^{\mathrm{T}} \mathrm{z}, \\
\bar{H}=\frac{1}{2} \overline{\mathbf{z}}^{\mathrm{T}} I \overline{\mathbf{z}},
\end{gathered}
$$

where

$$
\mathbf{z}=\left[\begin{array}{l}
q \\
p
\end{array}\right], \quad \overline{\mathbf{z}}=\left[\begin{array}{l}
Q \\
P
\end{array}\right], \quad \mathbf{b}=\left[\begin{array}{l}
f \\
0
\end{array}\right]
$$

the transformation is

where [5]

$$
\mathbf{z}=S \overline{\mathbf{z}}+\mathbf{r}
$$

$$
\begin{aligned}
& \dot{S}=J I S-S J I, \\
& \mathbf{t}=J \mathbf{r}+J \mathbf{b} .
\end{aligned}
$$


The matrix $J$ is the $2 \times 2$ symplectic matrix. There is no loss of generality in taking $S$ to be the identity. The solution of (3.9) is given by

$$
\mathbf{r}(t)=R(t) \mathbf{r}(0)+\int_{0}^{t} R(t) R^{-1}(\tau) J \mathbf{b}(\tau) d \tau,
$$

where $R(t)$ is the $2 \times 2$ matrix formed from the solution set of the homogeneous equation corresponding to (3.9). Explicitly,

$$
R(t)=\left[\begin{array}{cc}
\cos t & \sin t \\
-\sin t & \cos t
\end{array}\right]
$$

Again there is no loss of generality if $\mathbf{r}(0)$ is set at zero. The transformation relating the two Hamiltonians is then

where

$$
\left[\begin{array}{l}
q \\
p
\end{array}\right]=\left[\begin{array}{l}
Q \\
P
\end{array}\right]+\left[\begin{array}{c}
g(t) \\
-h(t)
\end{array}\right],
$$

$$
\begin{aligned}
& g(t)=\int_{0}^{t} \sin (\tau-t) f(\tau) d \tau, \\
& h(t)=\int_{0}^{t} \cos (\tau-t) f(\tau) d \tau .
\end{aligned}
$$

The two linear and three quadratic invariants for (3.2) are (compare with [8]):

$$
\left.\begin{array}{rl}
2 \phi_{1}(q, p, t) & =-\left\{(q-g)^{2}-(p+h)^{2}\right\} \sin 2 t-2(q-g)(p+h) \cos 2 t, \\
2 \phi_{2}(q, p, t) & =-\left\{(q-g)^{2}-(p+h)^{2}\right\} \cos 2 t+2(q-g)(p+h) \sin 2 t, \\
\phi_{3}(q, p, t) & =-(q-g) \sin t-(p+h) \cos t, \\
\phi_{4}(q, p, t) & =(q-g) \cos t-(p+h) \sin t, \\
2 \phi_{5}(q, p, t) & =(p+h)^{2}+(q-g)^{2} .
\end{array}\right\}
$$

The ordering adopted is that which has been used in recent papers $[10,13,15]$. From (2.2) and (2.10) it follows that the corresponding generators are

$$
\begin{aligned}
& G_{1}=\sin 2 t \frac{\partial}{\partial t}+\{(q-g) \cos 2 t-h \sin 2 t\} \frac{\partial}{\partial q} \\
& G_{2}=\cos 2 t \frac{\partial}{\partial t}-\{(q-g) \sin 2 t+h \cos 2 t\} \frac{\partial}{\partial q} \\
& G_{3}=\cos t \frac{\partial}{\partial q} \\
& G_{4}=\sin t \frac{\partial}{\partial q} \\
& G_{5}=\frac{\partial}{\partial t}-h \frac{\partial}{\partial q}
\end{aligned}
$$


These five generators have commutation relations which are appropriate for the five-parameter proper subgroup of $S l(3, R)$. They are

$$
\left.\begin{array}{l}
{\left[G_{1}, G_{2}\right]=-2 G_{5}, \quad\left[G_{5}, G_{1}\right]=2 G_{2}, \quad\left[G_{2}, G_{5}\right]=2 G_{1},} \\
{\left[G_{3}, G_{4}\right]=0,} \\
{\left[G_{3}, G_{1}\right]=\left[G_{2}, G_{4}\right]=\left[G_{5}, G_{4}\right]=G_{3},} \\
{\left[G_{1}, G_{4}\right]=\left[G_{3}, G_{5}\right]=\left[G_{2}, G_{3}\right]=G_{4} .}
\end{array}\right\}
$$

\section{Transformation of the generators}

The remaining three generators, which also constitute a proper subgroup of $S l(3, R)$, may be obtained by solving the partial differential equation (2.8) for $\xi(q, t)$ and $\eta(q, t)$. This has the disadvantage of including the derivation of the generators already obtained. Further, for this problem, the ordinary equations which emerge are coupled, which complicates the algebra. An alternative method, which has been used elsewhere [11], is to compare the expressions for $G_{1}$ to $G_{5}$ for the harmonic oscillator with those obtained in this case, for the forced oscillator. By noting the variations, an educated guess can be made as to the expressions for $G_{6}$ to $G_{7}$. The guess can be checked by substitution of $\xi$ and $\eta$ in (2.8). There is another method which may be applied in this problem. Before outlining it, the generators for the harmonic oscillator described by (3.3) are listed. They are

$$
\left.\begin{array}{l}
G_{1}\left(Q, t^{\prime}\right)=\sin 2 t^{\prime} \frac{\partial}{\partial t^{\prime}}+Q \cos 2 t^{\prime} \frac{\partial}{\partial Q}, \\
G_{2}\left(Q, t^{\prime}\right)=\cos 2 t^{\prime} \frac{\partial}{\partial t^{\prime}}-Q \sin 2 t^{\prime} \frac{\partial}{\partial Q}, \\
G_{3}\left(Q, t^{\prime}\right)=\cos t^{\prime} \frac{\partial}{\partial Q}, \\
G_{4}\left(Q, t^{\prime}\right)=\sin t^{\prime} \frac{\partial}{\partial Q}, \\
G_{5}\left(Q, t^{\prime}\right)=\frac{\partial}{\partial t^{\prime}}, \\
G_{6}\left(Q, t^{\prime}\right)=Q \frac{\partial}{\partial Q}, \\
G_{7}\left(Q, t^{\prime}\right)=Q \sin t^{\prime} \frac{\partial}{\partial t^{\prime}}+Q^{2} \cos t^{\prime} \frac{\partial}{\partial Q}, \\
G_{8}\left(Q, t^{\prime}\right)=Q \cos t^{\prime} \frac{\partial}{\partial t^{\prime}}-Q^{2} \sin t^{\prime} \frac{\partial}{\partial Q} .
\end{array}\right\}
$$


The transformation from $\bar{H}$ to $H$ may be written as

$$
\left.\begin{array}{l}
q=Q+g\left(t^{\prime}\right), \\
p=P-h\left(t^{\prime}\right), \\
t=t^{\prime},
\end{array}\right\}
$$

where the distinction is made between $t$ and $t^{\prime}$ for reasons which become apparent shortly. The transformation (4.2) is a point transformation and the second equation adds no further information to that contained in the other two. (Note that $d g\left(t^{\prime}\right) / d t^{\prime}=-h\left(t^{\prime}\right)$.) The differential operators in (4.1), namely $\partial / \partial t^{\prime}$ and $\partial / \partial Q$, take the following expression in the new coordinates,

$$
\left.\begin{array}{l}
\frac{\partial}{\partial t^{\prime}}=\frac{\partial t}{\partial t^{\prime}} \frac{\partial}{\partial t}+\frac{\partial q}{\partial t^{\prime}} \frac{\partial}{\partial q}=\frac{\partial}{\partial t}-h \frac{\partial}{\partial q}, \\
\frac{\partial}{\partial Q}=\frac{\partial t}{\partial Q} \frac{\partial}{\partial t}+\frac{\partial q}{\partial Q} \frac{\partial}{\partial q}=\frac{\partial}{\partial q} .
\end{array}\right\}
$$

If, in the first five equations in (4.1), $Q$ is replaced by $q-g, t^{\prime}$ by $t, \partial / \partial t^{\prime}$ by $\partial / \partial t-L \partial / \partial q$ and $\partial / \partial Q$ by $\partial / \partial q$, the operators are now in the form given in (3.16). The remaining generators for the forced oscillator are

$$
\left.\begin{array}{l}
G_{6}=(q-g) \frac{\partial}{\partial q}, \\
G_{7}=(q-g) \sin t \frac{\partial}{\partial t}+\left\{(q-g)^{2} \cos t-(q-g) h \sin t\right\} \frac{\partial}{\partial q}, \\
G_{8}=(q-g) \cos t \frac{\partial}{\partial t}-\left\{(q-g)^{2} \sin t+(q-g) h \cos t\right\} \frac{\partial}{\partial q} .
\end{array}\right\}
$$

It is simply a matter of algebra to verify that $G_{6}, G_{7}$ and $G_{8}$ do form a proper subgroup of $S L(3, R)$. The commutation relations are

$$
\left[G_{6}, G_{7}\right]=G_{7}, \quad\left[G_{6}, G_{8}\right]=G_{8}, \quad\left[G_{7}, G_{8}\right]=0 .
$$

The commutation relations between the generators of the two subgroups are also the standard ones, being

$$
\begin{aligned}
& {\left[G_{6}, G_{1}\right]=\left[G_{6}, G_{2}\right]=\left[G_{6}, G_{5}\right]=0,} \\
& {\left[G_{7}, G_{1}\right]=-G_{7},\left[G_{7}, G_{2}\right]=-G_{8},\left[G_{7}, G_{5}\right]=-G_{8},} \\
& {\left[G_{8}, G_{1}\right]=G_{8},\left[G_{8}, G_{2}\right]=-G_{7},\left[G_{8}, G_{5}\right]=G_{7},} \\
& {\left[G_{6}, G_{3}\right]=-G_{3},\left[G_{6}, G_{4}\right]=-G_{4},} \\
& {\left[G_{7}, G_{3}\right]=-\frac{1}{2}\left(G_{1}+3 G_{6}\right),\left[G_{8}, G_{4}\right]=\frac{1}{2}\left(-G_{1}+3 G_{6}\right),} \\
& {\left[G_{7}, G_{4}\right]=\frac{1}{2}\left(G_{2}-G_{5}\right),\left[G_{8}, G_{3}\right]=-\frac{1}{2}\left(G_{2}+G_{5}\right) .}
\end{aligned}
$$


Thus the complete symmetry group of the one-dimensional forced harmonic oscillator is $S l(3, R)$.

\section{Discussion}

The result obtained here for the forced oscillator applies equally well to an oscillator with a moving source of potential for it is essentially the same problem, having the same equation of motion. The transformation between the two Hamiltonians was of the form of identity plus a time-dependent displacement. Thus the symmetry group of any forced linear system will be the same as the corresponding unforced linear system. As the symmetry group $\operatorname{Sl}(3, R)$ is applicable to the free particle, attractive and repulsive (both time-independent and timedependent) oscillators, it is applicable to the case of a particle moving under the influence of a coordinate-free force and to the forced attractive and repulsive oscillator. It is also applicable to motion relative to frames of reference in rectilinear motion (the equivalent for the oscillators to a moving source of potential). It might be noted that this is independent, in the case of oscillators, of whether the forcing term (or source movement) is resonant or not.

For $n$-dimensional systems without coupling, damping or rotating axes, the corresponding result is that the symmetry group is $S l(n+2, R)$. The excluded cases await further investigation.

The use of point transformations may make the investigation of the symmetries of other systems easier. To take the example of the time-dependent harmonic oscillator which has been studied in other ways $[10,15]$, the Hamiltonians

$$
H=\frac{1}{2}\left\{p^{2}+\omega^{2}(t) q^{2}\right\}
$$

and

$$
\bar{H}=\rho^{-2}\left\{P^{2}+Q^{2}\right\}
$$

are related by the point transformation

$$
\left[\begin{array}{l}
q \\
p
\end{array}\right]=\left[\begin{array}{ll}
1 / \rho & 0 \\
-\dot{\rho} & \rho
\end{array}\right]\left[\begin{array}{l}
Q \\
P
\end{array}\right]
$$

where $\rho(t)$ is any solution of

$$
\ddot{\rho}+\omega^{2}(t) \rho=1 /\left(\rho^{3}\right) .
$$

Hence the symmetries of the systems corresponding to $H$ and $\bar{H}$ are the same. The change of time variable from $t$ to $W$, where

$$
W=\int_{t_{0}}^{t} \rho^{-2} d t^{\prime}
$$


makes (5.2) the equivalent to the time-independent harmonic oscillator which possesses $S l(3, R)$ symmetry. The problem of determining whether a given dynamical system possesses this symmetry is reduced to finding a point transformation relating it to a system which does.

\section{Acknowledgements}

The writer wishes to thank Professor C. J. Eliezer, who introduced him to the subject of dynamical symmetries some years ago, and G. E. Prince for stimulating discussions on the subject of Lie groups.

\section{References}

[1] R. L. Anderson and S. M. Davison, "A generalization of Lie's 'counting' theorem for second-order differential equations", J. Math. Anal. Applic. 48 (1974), 301-315.

[2] G. W. Bluman and J. D. Cole, Similarity methods for differential equations (Springer Verlag, New York, 1974).

[3] N. J. Günther and P. G. L. Leach, "Generalized invariants for the time-dependent harmonic oscillator", J. Math. Phys. 18 (1977), 572-576.

[4] J. M. Jauch and E. L. Hill, "On the problem of degeneracy in quantum mechanics", Phys. Rev. 57 (1940), 641-645; D. M. Fradkin, "Three dimensional isotropic oscillator and SU(3)", Amer. J. Phys. 33 (1965), 207-211.

[5] P. G. L. Leach, "On the theory of time-dependent linear canonical transformations as applied to Hamiltonians of the harmonic oscillator type", J. Math. Phys. 18 (1977), 1608-1611.

[6] P. G. L. Leach, "Invariants and wave-functions for some time-dependent harmonic oscillator type Hamiltonians", J. Math. Phys. 18 (1977), 1902-1907.

[7] P. G. L. Leach, "Quadratic Hamiltonians, quadratic invariants and the symmetry groups, $S U(n)$ ", J. Math. Phys. 19 (1978), 446-451.

[8] P. G. L. Leach, "The invariants of quadratic Hamiltonians, I: Linear and quadratic invariants for the time-independent isotropic harmonic oscillator"' (Preprint, Department of Applied Mathematics, La Trobe University, March 1978).

[9] P. G. L. Leach, "Quadratic Hamiltonians: The four classes of quadratic invariants, their interrelations and symmetries" (Preprint, Department of Applied Mathematics, La Trobe University, September 1978).

[10] P. G. L. Leach, "The complete symmetry group of the one-dimensional time-dependent harmonic oscillator"' (Preprint, Department of Applied Mathematics, La Trobe University, December 1978).

[11] P. G. L. Leach, " $S l(3, R)$ and the repulsive oscillator" (Preprint, Department of Applied Mathematics, La Trobe University, May 1979).

[12] H. R. Lewis, Jr., "Class of exact invariants for classical and quantum time-dependent harmonic oscillators", J. Math. Phys. 9 (1968), 1976-1986.

[13] M. Lutzky, "Symmetry groups and conserved quantities for the harmonic oscillator", $J$. Phys. A 11 (1978), 249-258.

[14] M. Lutzky, "Dynamical symmetries and conserved quantities", J. Phys. A 12 (1979),

[15] G. E. Prince and C. J. Eliezer, "Symmetries of the time-dependent $N$-dimensional oscillator" (Preprint, Department of Applied Mathematics, La Trobe University, March 1979). 
[16] G. E. Prince, private communication, May, 1979.

[17] C. E. Wulfman and B. G. Wybourne, "The Lie group of Newton's and Lagrange's equations for the harmonic oscillator", J. Phys. A 9 (1976), 507-518.

Department of Applied Mathematics

La Trobe University

Bundoora 3083

Australia 